\title{
Study on evaluation of gamma oryzanol of germinated brown rice by near infrared spectroscopy
}

\author{
Kannapot Kaewsorn and Panmanas Sirisomboon* \\ Curriculum of Agricultural Engineering \\ Department of Mechanical Engineering \\ Faculty of Engineering \\ King Mongkut's Institute of Technology Ladkrabang \\ Chalongkrung Road, Ladkrabang \\ Bangkok 10520, Thailand \\ *kspanman@kmitl.ac.th
}

Accepted 25 October 2013

Published 19 December 2013

\begin{abstract}
Germinated brown rice (GBR) is rich in gamma oryzanol which increase its consumption popularity, particularly in the health food market. The objective of this research was to apply the near infrared spectroscopy (NIRS) for evaluation of gamma oryzanol of the germinated brown rice. The germinated brown rice samples were prepared from germinated rough rice (soaked for 24 and $48 \mathrm{~h}$, incubated for 0 , $6,12,18,24,30$ and $36 \mathrm{~h}$ ) and purchased from local supermarkets. The germinated brown rice samples were subjected to NIR scanning before the evaluation of gamma oryzanol by using partial extraction methodology. The prediction model was established by partial least square regression (PLSR) and validated by full cross validation method. The NIRS model established from various varieties of germinated brown rice bought from different markets by first derivatives+vector normalization pretreated spectra showed the optimal prediction with the correlation of determination $\left(R^{2}\right)$, root mean squared error of cross validation (RMSECV) and bias of $0.934,8.84 \times 10^{-5} \mathrm{mg} / 100 \mathrm{~g}$ dry matter and $1.06 \times 10^{-5} \mathrm{mg} / 100 \mathrm{~g}$ dry matter, respectively. This is the first report on the application of NIRS in the evaluation of gamma oryzanol of the germinated brown rice. This information is very useful to the germinated brown rice production factory and consumers.
\end{abstract}

Keywords: Germinated brown rice; gamma oryzanol; near infrared spectroscopy.

\section{Introduction}

Germinated brown rice (GBR) contains more chemicals and functional components including gammaogyzanol and gamma aminobutyric acid than the ungerminated brown rice. ${ }^{1}$ In the rice bran, there is vitamin $\mathrm{E}$ ( $\alpha$-tocopherol and tocotrienol) and gamma-ogyzanol. ${ }^{2}$

Gamma oryzanol is a naturally occurring component in rice bran and germ. It is a complex mixture of furalate, esterified with sterols or triterpene

This is an Open Access article published by World Scientific Publishing Company. It is distributed under the terms of the Creative Commons Attribution 3.0 (CC-BY) License. Further distribution of this work is permitted, provided the original work is properly cited. 
alcohols. ${ }^{3}$ Gamma oryzanol in rice bran was about 13 to 20 times $(\mathrm{w} / \mathrm{w})$ greater than tocopherol and tocotrienols. ${ }^{4}$

Gamma oryzanol was shown to be able to reduce cholesterol absorption. ${ }^{5}$ It was appropriate for the treatment of the inflammatory process ${ }^{6}$ and it could inhibit linoleic acid and cholesterol oxidation. ${ }^{7,8}$ In addition, it is a potential antioxidant for food, pharmaceutical and cosmetic industries. ${ }^{9,10}$

From Kim et al., ${ }^{1}$ the rough rice of Ilpumbyeo variety was soaked in water at $15^{\circ} \mathrm{C}$ for three days and the results showed that the gamma oryzanol content of different parts (rough rice, hull, brown rice and sprout) was increased by 1.13 and 1.20 fold in germinated rough rice and GBR, respectively.

The near infrared spectroscopy (NIRS) technique can provide rapid results in seconds or continuously online, rather than in hours or days, with an accuracy and reproducibility equivalent to most reference methods and other advantages of NIR include its low cost per test, low labor costs, no required chemicals to purchase or dispose of, great flexibility in sample presentation and the capability of testing many constituents simultaneously. ${ }^{11}$

NIRS has been applied to many types of agricultural products. Researchers have utilized NIRS to analyze chemical components of rice, such as amylose content, protein content, lipid content, ${ }^{12-16}$ fatty acid of rough rice, ${ }^{17}$ lipid content of milled rice $^{18}$ and amino acid in brown rice. ${ }^{19}$

The objective of this research was to apply NIRS for evaluation of gamma oryzanol of the GBR.

\section{Materials and Methods}

\subsection{Rice samples}

The GBR samples were prepared by a factory of P.J. Brand germinated rough rice in Chonburi Province, Thailand and purchased from local markets in Thailand.

\subsubsection{Preparation of $G B R$ by a factory}

Rough rice of Oryza sativa L., cultivar Khao Dawk Mali 105 (KDML 105) was collected from a field of P.J. Brand germinated rough rice factory in Chonburi Province, Thailand. Rough rice was soaked in water at room temperature for 24 or $48 \mathrm{~h}$. The water was changed every $4 \mathrm{~h}$ and drained at the end of soaking. The rice was kept in polypropylene sag for incubating at seven different duration $(0,6,12$, $18,24,30$ and $36 \mathrm{~h}$ ) to obtain the germinated rough rice (GRR). The GRR was dried using fluidizedbed technique by superheated steam which would reduce the moisture content of the rice to around $19 \% \mathrm{wb}$. Then the rice was spread on a screen for $3 \mathrm{~h}$ at room temperature and the moisture content would reduce to about $13-14 \%$ wb. After that the GRR sample was dehusked before the experiment and it would be called "GBR-Oryzanol adjusted" in this paper. The rough rice sample used was $10 \mathrm{~kg}$ for 1 treatment. There was one control condition and 15 treatments with 4 replicates resulted in 68 samples in total. In addition, there were 20 samples from the rough rice sample prepared by the commercial production condition (soaked for $48 \mathrm{~h}$ and incubated for $24 \mathrm{~h}$ ), hence, 88 samples from a factory.

\subsubsection{Germinated brown rice from local markets in Thailand (MGBR)}

All GBR of different brands were purchased from local markets in Thailand and stored in the laboratory at room temperature. These type of samples would be called "market-germinated brown rice (MGBR)" in this paper. Each brand bought was separated into four subsamples. There were 16 brands for KDML 105 variety (68 samples, MGBRKDML 105) and 16 brands for other varieties such as Red Jasmine rice, Hom Nil, Kam Doi Muser, RD6, Kam Doi Mae jam, Tang Doi, Khao Doi (short grain), Khao Na (short grain), Dok Pradu, Dang Pa Tam, Hom Dang Sukhothai, Hom Sin Lek, Hom Dam Pathumrat, Nheaw Kam and Nheaw RD6 (68 samples, MGBR-various varieties). Hence, 136 samples from local markets.

\subsection{Near infrared spectroscopy experiment}

\subsubsection{Sample scanning}

Each GBR sample was poured from the vacuum bag into the quartz-sampling cup of a rotary diffuse reflectance holder (Bruker Ltd., Germany). NIRS was measured with Fourier transform near infrared (FT-NIR) spectrometer (Bruker Ltd., Germany) in reflection mode on $12500-4000 \mathrm{~cm}^{-1}(800-2500 \mathrm{~nm})$. Each rice sample was scanned 64 times at a resolution of $16 \mathrm{~cm}^{-1}$. The scan results were averaged and 
recorded in absorption mode $(\log 1 / R)$ for each sample. The quartz-sampling cup was cleaned with a vacuum cleaner prior to subsequent use.

\subsubsection{Spectrum pre-treatment and NIRS model establishment}

The NIRS models for predicting the gamma oryzanol content of GBR were established using the partial least squares regression (PLSR). The software for multivariate analysis (OPUS, v. 7.0.129) was used in both spectrum pre-processing and model development. The NIR spectra used to model development were not pre-processing spectra or preprocessing spectra using any of the following methods: constant offset elimination, straight line subtraction, vector normalization (SNV), min-max normalization, multiplicative scatter correction (MSC), first derivatives, second derivatives, first derivatives + straight line subtraction, first derivatives+SNV and first derivatives+MSC.

After model development, the outlier samples were identified by the software and they were removed.

The optimum model was selected by coefficients of determination $\left(R^{2}\right)$, root mean squared error of cross validation (RMSECV), ratio of standard error of validation to the standard deviation (RPD) and bias.

\subsection{Analysis of gamma oryzanol}

The gamma oryzanol content was determined by following the method of Lilitchan et al. ${ }^{20}$ by using partial extraction methodology. After scanning, $50 \mathrm{~g}$ of GBR was ground by cereal grinder (Oku San No, Malaysia). The ground sample was weighed into two identical vials ( $0.5 \mathrm{~g}$ each) and extracted with n-hexane using different volumes (4 and $8 \mathrm{~mL}$ ) by vigorous agitation on the vortex mixer for $1 \mathrm{~min}$, at room temperature. The mixture was centrifuged at $2500 \mathrm{rpm}$ for $10 \mathrm{~min}$. The absorbance of two supernatants was measured at $314 \mathrm{~nm}$ using a GENESYS 10S UV-Vis spectrophotometer (USA). The gamma oryzanol content in the extracts were quantified against the standard curve. Total gamma oryzanol was calculated by solving two simultaneous equations:

$$
k=\left(\frac{x}{v}\right)\left(\frac{w}{y-x}\right)
$$

where:

$k=\mathrm{A}$ constant

$x=$ The amount of gamma oryzanol in the extract $(\mathrm{g})$,

$y=$ The amount of gamma oryzanol in bran,

$v=$ The volume used for extraction $(\mathrm{mL})$,

$w=$ The weight of bran used for extraction.

There are two unknowns ( $k$ and $y$ ), thus the extraction of two identical samples with different volumes of solvent are necessary. In order to simplify the analysis and the calculation, the solvent used for the second extraction is doubled $\left(V_{2}=2 V_{1}\right)$ and the amount of solute in the two extracts are $x_{1}$ and $x_{2}$. Although the $k$ value for solid extraction is slightly varied, as the amount of solvent used for extractions is different, it is assumed that the change does not affect the accuracy of this study. Thus, by assuming $k_{1}=k_{2}$, the total amount of gamma oryzanol $(y)$ can be calculated:

$$
y=\frac{x_{1} x_{2}}{2 x_{1}-x_{2}} .
$$

\subsection{Overall precision test}

The overall precision, i.e., reproducibility, of the reference test was determined by selecting samples, 10 as blind duplicates ( 5 pairs) along with the normal experiment. The reproducibility is the standard deviation (SD) of the differences between the values of blind duplicates. In addition, the repeatability of the reference test was determined by the SD of the differences between the values of another 10 as duplicates (5 pairs) which were not blind samples.

\section{Result and Discussion}

The six groups of GBR samples included GBROryzanol adjusted, MGBR, MGBR- KDML 105, MGBR-various varieties, GBR-Oryzanol adjusted+ MGBR-KDML 105 and GBR-Oryzanol adjusted+ MGBR that were used for model development. In the outlier identification process, there were $4,3,4$, 4, 5 and 6 outliers that were removed, respectively. The minimum (Min), maximum (Max), mean, and SD values of gamma oryzanol content of GBR of different sample sets are shown in Table 1. The range of gamma oryzanol content was from $6.06 \times$ $10^{-4}$ to $3.27 \times 10^{-3} \mathrm{mg} / 100 \mathrm{~g}$ dry matter.

The statistics of prediction performance of the PLS model for gamma oryzanol content of GBR 
Table 1. Minimum (Min), maximum (Max), mean and SD of gamma oryzanol content of the GBR.

\begin{tabular}{lcccc}
\hline Parameter & $\begin{array}{c}\text { Number of } \\
\text { samples }\end{array}$ & $\begin{array}{c}\text { Min } \\
(\mathrm{mg} / 100 \mathrm{~g} \text { dry matter })\end{array}$ & $\begin{array}{c}\text { Max } \\
(\mathrm{mg} / 100 \mathrm{~g} \text { dry matter })\end{array}$ & $\begin{array}{c}\text { Mean } \\
(\mathrm{mg} / 100 \mathrm{~g} \text { dry matter })\end{array}$ \\
\hline SBR-Oryzanol adjusted & 84 & $9.87 \times 10^{-4}$ & $2.11 \times 10^{-3}$ & $1.44 \times 10^{-3}$ \\
MGBR & 133 & $6.06 \times 10^{-4}$ & $2.23 \times 10^{-3}$ & $1.24 \times 10^{-3}$ \\
MGBR-KDML 105 & 64 & $6.06 \times 10^{-4}$ & $2.23 \times 10^{-3}$ & $1.31 \times 10^{-3}$ \\
MGBR-various varieties & 64 & $6.87 \times 10^{-4}$ & $1.87 \times 10^{-3}$ & $4.32 \times 10^{-4}$ \\
$\begin{array}{l}\text { GBR-Oryzanol adjusted } \\
\quad \text { +MGBR-KDML 105 }\end{array}$ & 151 & $6.06 \times 10^{-4}$ & $2.23 \times 10^{-3}$ & $1.38 \times 10^{-3}$ \\
$\begin{array}{l}\text { GBR-Oryzanol } \\
\quad \text { adjusted+MGBR }\end{array}$ & 218 & $6.06 \times 10^{-4}$ & $3.27 \times 10^{-3}$ & $3.59 \times 10^{-4}$ \\
\hline
\end{tabular}

Table 2. Statistics of prediction of gamma oryzanol content of GBR by PLS models.

\begin{tabular}{|c|c|c|c|c|c|c|c|}
\hline Parameter & Pre-processing & Range & PLS factor & $R^{2}$ & RMSECV & $\mathrm{RPD}$ & Bias \\
\hline GBR-Oryzanol adjusted & First derivatives + MSC & $\begin{array}{c}9403.8-7498.3 \\
4605.4-4242.9\end{array}$ & 4 & 0.610 & $1.78 \times 10^{-4}$ & 1.60 & $7.12 \times 10^{-7}$ \\
\hline MGBR & Second derivatives & $\begin{array}{l}9403.8-7498.3 \\
6102-5168.6\end{array}$ & 8 & 0.535 & $2.79 \times 10^{-4}$ & 1.47 & $-3.40 \times 10^{-6}$ \\
\hline MGBR-KDML 105 & SNV & $\begin{array}{c}8454.9-7498.3 \\
6102-4597.7\end{array}$ & 9 & 0.768 & $2.06 \times 10^{-4}$ & 2.08 & $-6.09 \times 10^{-6}$ \\
\hline $\begin{array}{l}\text { MGBR-various } \\
\text { varieties }\end{array}$ & First derivatives + SNV & $\begin{array}{c}9403.8-7498.3 \\
6102-4597.7\end{array}$ & 10 & 0.934 & $0.88 \times 10^{-4}$ & 3.91 & $1.06 \times 10^{-5}$ \\
\hline $\begin{array}{c}\text { GBR-Oryzanol adjusted } \\
\text { +MGBR-KDML } 105\end{array}$ & MSC & $6102-4597.7$ & 9 & 0.482 & $2.63 \times 10^{-4}$ & 1.39 & $-7.23 \times 10^{-6}$ \\
\hline $\begin{array}{l}\text { GBR-Oryzanol adjusted } \\
\quad+\text { MGBR }\end{array}$ & $\begin{array}{l}\text { First derivatives+straight } \\
\quad \text { line subtraction }\end{array}$ & $6102-4597.7$ & 9 & 0.536 & $2.53 \times 10^{-4}$ & 1.47 & $2.23 \times 10^{-6}$ \\
\hline
\end{tabular}

$R^{2}$ - Coefficient of determination; RMSECV - Root mean squared error of cross validation; RPD — Ratio of standard error of validation to the SD; Bias - Average error of prediction.

developed using six different groups of samples is shown in Table 2. The optimum models for gamma oryzanol content evaluation of MGBR-various varieties derived from 10 PLS factors from the spectra in the range of $9403.8-7498.3 \mathrm{~cm}^{-1}$ and $6102-$ $4597.7 \mathrm{~cm}^{-1}$ that were pre-processed by the first derivatives+SNV methods showed the coefficients of determination $\left(R^{2}\right)$, root mean square error of cross validation (RMSECV), ratio of standard error of validation to the standard deviation (RPD) and bias of $0.934,0.88 \times 10^{-4} \mathrm{mg} / 100 \mathrm{~g}$ dry matter, 3.91 and $1.06 \times 10^{-5} \mathrm{mg} / 100 \mathrm{~g}$ dry matter, respectively.

Williams $^{22}$ indicated that $R^{2}$ of $0.92-0.96$ implied that the model was usable in most applications, including quality assurance.

The scatter plots (reference data $(X)$ with prediction data $(Y)$ ) of gamma oryzanol content are shown in Fig. 1.

Furthermore, the prediction of gamma oryzanol content of MGBR-KDML 105 provided $R^{2}$ value in prediction which were high $R^{2}$ values $(0.768)$ and low bias $\left(-6.09 \times 10^{-6} \mathrm{mg} / 100 \mathrm{~g}\right.$ dry matter $)$. For the other groups, the prediction of gamma oryzanol content showed low $R^{2}(0.535-0.610)$.

Williams ${ }^{22}$ indicated that $R^{2}$ of $0.66-0.81 \mathrm{im}-$ plied that the model was okay for screening and some other "approximate" calibration.

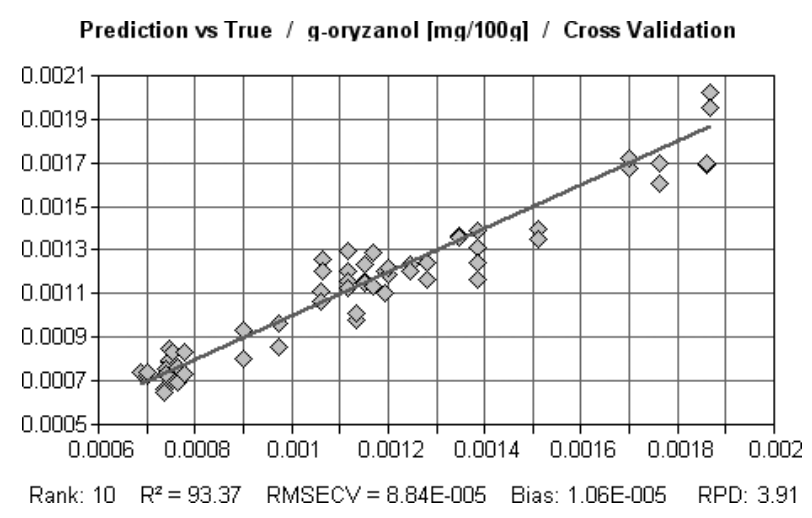

Fig. 1. Comparison of gamma oryzanol content of MGBRvarious varieties between NIRS prediction and reference lab. 


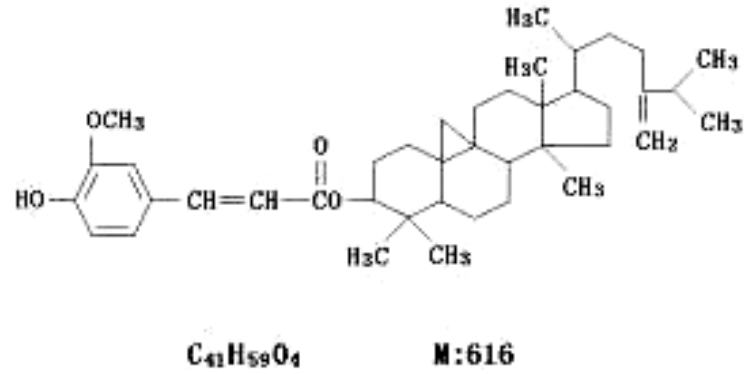

Fig. 2. Chemical structure of gamma oryzanol. ${ }^{23}$

Gamma oryzanol is a group of ferulic acid esters of phytosterols and triterpene alcohols. ${ }^{21}$ The chemical structure of gamma oryzanol is shown in Fig. 2.

Figure 3 shows the regression coefficient plot of gamma oryzanol content of MGBR-various varieties group. It could be seen that the high regression coefficient was illustrated at the absorption bands of $\mathbf{8 7 1 4}, \mathbf{8 3 7 8}, 6090,5936,5311, \mathbf{5 1 2 2}, 5048,4912$, 4815,4728 and $4633 \mathrm{~cm}^{-1}(\mathbf{1 1 4 8}, \mathbf{1 1 9 4}, 1642,1685$,

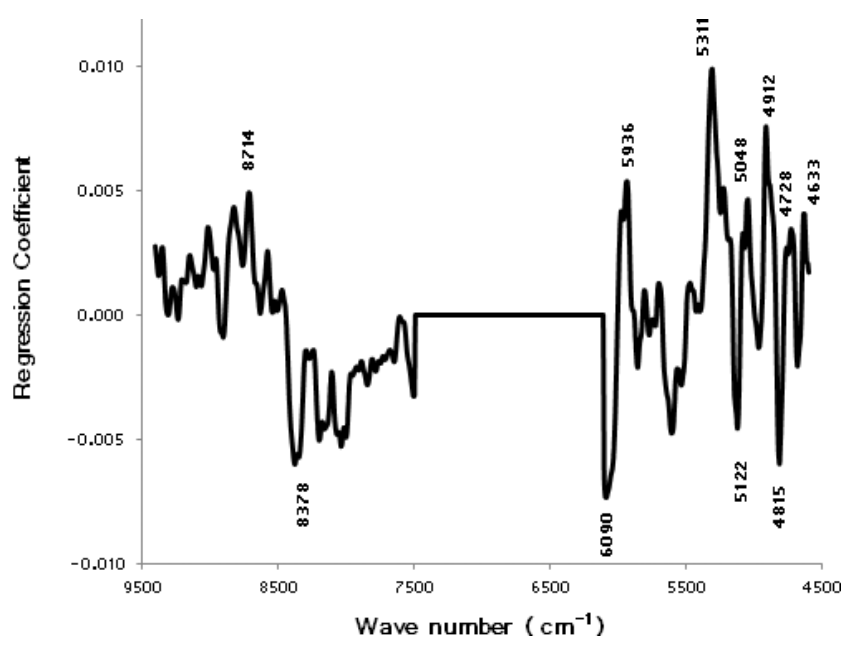

Fig. 3. Regression coefficient plot of gamma oryzanol content of MGBR-various varieties.
1883, 1952, 1981, 2036, 2077, 2115 and $2158 \mathrm{~nm})$. The vibration at $8714 \mathrm{~cm}^{-1}(1148 \mathrm{~nm})$ and 8378 (1194 nm) might be due to the combination vibration of $\mathrm{C}-\mathrm{H}$ stretch second overtone of $\mathrm{CH}_{3}$. According to Osborne and Fearn, ${ }^{22}$ the absorbance bands of the bond vibration are at 1152 and $1195 \mathrm{~nm}$. Furthermore, the vibration at $5122 \mathrm{~cm}^{-1}(1952 \mathrm{~nm})$ might be due to the combination vibration of $\mathrm{C}=\mathrm{O}$ stretch second overtone of $-\mathrm{CO}_{2} \mathrm{R}$. The bond vibration was indicated by Osborne and Fearn ${ }^{24}$ at $1950 \mathrm{~nm}$. These bonds are in the gamma oryzanol chemical structure. Therefore, the vibration of oryzanol was effected and included in the model.

Table 3 shows the overall precision of reference testing for gamma oryzanol of GBR samples. The SD of differences of all duplicates, i.e., repeatability was $0.25 \times 10^{-3} \mathrm{mg} / 100 \mathrm{~g}$ dry matter and the SD of differences of blind duplicates, i.e., reproducibility was $0.48 \times 10^{-3} \mathrm{mg} / 100 \mathrm{~g}$ dry matter.

\section{Conclusion}

From the study, the NIRS of GBR could be an alternative technique to evaluate the gamma oryzanol content of various varieties of market-germinated brown rice (MGBR-various varieties) because the model was usable in most applications, including quality assurance. Future work should be focused on the real application of the models generated in this study to predict the gamma oryzanol of samples drawn from the product of different factories and labeled it on the packages.

\section{Acknowledgment}

The authors would like to express their gratitude to National Research Council of Thailand and King

Table 3. Repeatability and reproducibility of reference test for gamma oryzanol of GBR. (mg/100g dry matter).

\begin{tabular}{|c|c|c|c|c|c|c|c|}
\hline \multicolumn{4}{|c|}{ Repeatability SD } & \multicolumn{4}{|c|}{ Reproducibility SD } \\
\hline Sample number & Duplicate.a & Duplicate.b & Diff. $a-b$ & Sample number & Duplicate.a & Duplicate.b & Diff. $a-b$ \\
\hline 5,17 & $1.29 \times 10^{-3}$ & $1.55 \times 10^{-3}$ & $-0.26 \times 10^{-3}$ & 11,18 & $1.24 \times 10^{-3}$ & $1.23 \times 10^{-3}$ & $0.01 \times 10^{-3}$ \\
\hline 24,33 & $1.19 \times 10^{-3}$ & $0.79 \times 10^{-3}$ & $0.40 \times 10^{-3}$ & 28,34 & $1.55 \times 10^{-3}$ & $0.86 \times 10^{-3}$ & $0.69 \times 10^{-3}$ \\
\hline 32,51 & $1.14 \times 10^{-3}$ & $1.24 \times 10^{-3}$ & $-0.09 \times 10^{-3}$ & 38,52 & $1.48 \times 10^{-3}$ & $2.07 \times 10^{-3}$ & $-0.59 \times 10^{-3}$ \\
\hline 59,63 & $1.95 \times 10^{-3}$ & $1.95 \times 10^{-3}$ & $1.62 \times 10^{-6}$ & 53,64 & $1.72 \times 10^{-3}$ & $1.38 \times 10^{-3}$ & $0.34 \times 10^{-3}$ \\
\hline \multirow[t]{3}{*}{75,87} & $1.51 \times 10^{-3}$ & $1.66 \times 10^{-3}$ & $-0.15 \times 10^{-3}$ & 79,88 & $1.06 \times 10^{-3}$ & $1.13 \times 10^{-3}$ & $-0.07 \times 10^{-3}$ \\
\hline & & $\mathrm{SD}$ & $0.25 \times 10^{-3}$ & & & $\mathrm{SD}$ & $0.48 \times 10^{-3}$ \\
\hline & & Mean & $-2.06 \times 10^{-5}$ & & & Mean & $0.07 \times 10^{-3}$ \\
\hline
\end{tabular}


Mongkut's Institute of Technology Ladkrabang, Thailand for financial support.

\section{References}

1. H. Y. Kim, I. G. Hwang, T. M. Kim, K. S. Woo, D. S. Park, J. H. Kim, D. J. Kim, J. Lee, Y. R. Lee, H. S. Jeong, "Chemical and functional components in different part of rough rice (Oryza sativa L.) before and after germination," J. Food Chem. 134, 288-293 (2012).

2. X. Zhimin, H. Na, G. J. Samuel, "Antioxidant activity of tocopherols, tocotrienol, and $\gamma$-ogyzanol component from rice bran against cholesterol oxidation accelerated by 2,20-azobis (2-methylpropionamidine) dihydrochloride," J. Agric. Food Chem. 49, 2077-2081 (2001).

3. E. J. Roger, S. M. Rice, R. J. Nicolosi, D. R. Carpenter, C. A. McClelland, L. J. Romanczyk, "Identification and quantitation of $\gamma$-oryzanol components and simultaneous assessment of tocols in rice bran oil," J. Am. Oil Chem. Soc. 70, 301-303 (1993).

4. C. J. Bergman, Z. Xu, "Genotype and environment effects on tocopherol, tocotrienols and gamma oryzanol of southern US rice," Cereal Chem. 80, 446449 (2003).

5. N. Rong, L. M. Ausman, R. J. Nicolosi, "Oryzanol decreases cholesterol absorbtion and aortic fatty streak in hamsters," Lipids 32, 303-309 (1997).

6. T. Akihisa, K. Yasukawa, M. Yamaura, M. Ukiya, Y. Kimura, N. Shimisu, K. Arai, "Triterpene alcohol and sterol ferulates from rice bran and their antiinflammatory effects," J. Agric. Food Chem. 48, 2313-2319 (2000).

7. Z. Xu, J. S. Godber, "Antioxidant activities of major components of $\gamma$-oryzanol from rice bran using a linoleic acid model," J. Am. Oil Chem. Soc. 78, 645-649 (2001).

8. Z. Xu, J. S. Godber, "Antioxidant activities of tocopherol, tocotrienols and $\gamma$-oryzanol components from rice bran against cholesterol oxidation accelerated by 2,2'-Azobis (2-methylpro-piionamidine) dihydrochlorride," J. Agric. Food Chem. 49, 20772081 (2001).

9. J. N. Nanua, J. U. McGregor, J. S. Godber, "Influence of high-oryzanol rice bran oil on the oxidative stability of whole milk powder," J. Dairy Sci. 83, 2426-2431 (2000).

10. S. Iqbal, M. I. Bhanger, F. Anwar, "Antioxidant properties and components of some commercially available varieties of rice bran in Pakistan," Food Chem. 93, 265-272 (2005).

11. P. Sirisomboon, A. Kaewkuptong, P. Williams, "Feasibility study on the evaluation of the dry rubber content of field and concentrated latex of Para rubber by diffuse reflectance near infrared spectroscopy," J. Near Infrared Spectros. 21, 81-88 (2013).

12. R. K. Chang, W. Y. Zhang, J. Cui, Y. Z. Wang, Y. Wei, Y. Liu, "Research of rice-quality based on computer vision and near infrared spectroscopy," Computer and Computing Technologies in Agriculture III, D. Li, C. Zhao, Eds., CCTA 2009, IFIP Advances in Information and Communication Technology, Vol. 317, pp. 523-531, Springer-Verlag Berlin Heidelberg (2010).

13. E. T. Champagne, K. L. Bett-Garber, C. C. Grimm, A. M. McClung, K. A. Moldenhauer, S. Linscombe, K. S. McKenzie, F. E. Barton, "Near infrared reflectance analysis for prediction of cooked rice texture," Cereal Chem. 78(3), 358-362 (2001).

14. J. S. Bao, Y. Z. Cai, H. Corke, "Prediction of rice starch quality parameters by near-infrared reflectance spectroscopy," J. Food Sci. 66, 936-939 (2001).

15. N. Shimizu, J. Katsura, T. Yanagisawa, S. Inoue, R. P. Withey, I. A. Cowe et al., "Determination of apparent amylase content in Japanese milled rice using near-infrared transmittance spectroscopy," Food Sci. Technol. Res. 5(4), 337-342 (1999).

16. Q. Y. Shu, D. X. Wu, Y. M. Xia, M. W. Gao, A. McClung, "Analysis of grain quality characters in small ground brown rice samples by near infrared reflectance spectroscopy," Sci. Agric. Sin. 32, 92-97 (1999).

17. W.S. Li, J. T. Show, "Determining the fat acidity of rough rice by near-infrared reflectance spectroscopy," Cereal Chem. 74(5), 556-560 (1997).

18. H. Chen, B. P. Marks, T. J. Siebenmorgen, "Qualifying surface lipid content of milled rice via visible/ near-infrared spectroscopy," Cereal Chem. 74(6), 826-831 (1997).

19. B. Zhang, Z. Q. Rong, Y. Shi, J. G. Wu, C. H. Shi, "Prediction of amino acid composition in brown rice using different sample status by near-infrared reflectance spectroscopy," Food Chem. 127, 275-281 (2011).

20. S. Lilitchan, C. Tangprawat, K. Aryusuk, S. Krisnangkura, S. Chokmoh, K. Krisnangkura, "Partial extraction method for rapid analysis of total lipids and $\gamma$-oryzanol contents in rice bran," J. Food Chem. 106, 752-759 (2008).

21. R. Cheruvanky, Phytochemical products: Rice bran, Phytochemical Functional Foods, I. T. Jhonson 
and G. Williamson, Eds., pp. 347-376. CRC Press; Woodhead Publishing, Boca Raton, FL, Cambridge (2003).

22. P. Williams, Near-infrared Technology Getting the Best Out of Light, 5th Edition. A Short Course in the Practical Implementation of Near-Infrared Spectroscopy for the User, PDK Grain, Nanaimo, Canada (2007).
23. LookChem, Molecular Structure: Gamma oryzanol, Available at http://fgsbio.en.alibaba.com/product/ 512184895-209822355/Top_Quality_font_b_Gamma_ b_font_Oryzanol_CAS_No_11042_64_1_.html.

24. B. G. Osborne, T. Fearn, Near Infrared Spectroscopy in Food Analysis, p. 117, Longman Science \& Technical, UK (1986). 